\title{
E-cadherin regulates biological behaviors of neural stem cells and promotes motor function recovery following spinal cord injury
}

\author{
DONG CHEN ${ }^{*}$, SIYUAN HU*, JIE LIU and SHAOHUA LI \\ Department of Orthopedics, Shanghai Tenth People's Hospital, \\ Tongji University School of Medicine, Shanghai 200072, P.R. China
}

Received April 9, 2018; Accepted November 26, 2018

DOI: $10.3892 /$ etm.2019.7176

\begin{abstract}
Stem cell-based repair strategies for spinal cord injury (SCI) are a highly studied area of research. Multiple gene-modified stem cells have been transplanted into SCI models, in the hope of generating more neurons to repair a damaged nervous system. However, the results are not always successful, as the grafted cells may be unable to survive in the injured spinal cord. E-cadherin, a transmembrane adhesion protein, has been identified as an epithelial-to-mesenchymal transition marker and is vital for morphological structure maintenance and the functional integrity of epithelial cells. At present, few studies have examined the association between E-cadherin and neural stem cells (NSCs). The present study investigated the expression of E-cadherin in subcultured NSCs and differentiated NSCs. Furthermore, the effect of E-cadherin on NSC viability, migration, differentiation and neurosphere formation was assessed. An in vivo study was used to assess the long-term survival of grafted NSCs. Additionally, the protective effect of E-cadherin on SCI was assessed by analyzing tissue repair, Basso Mouse Scale scores and the expression of inflammatory cytokines. The results of the present study suggested that E-cadherin was able to promote NSC viability and neurosphere formation; however, it had no significant effect on NSC differentiation. To conclude, grafted NSCs with highly expressed E-cadherin facilitated motor function recovery following SCI by reducing the release of inflammatory cytokines.
\end{abstract}

\section{Introduction}

Spinal cord injury (SCI) is the leading cause of paralysis, resulting in a loss of function below the injury level (1). Unlike

Correspondence to: Dr Shaohua Li, Department of Orthopedics, Shanghai Tenth People's Hospital, Tongji University School of Medicine, 301 Yanchang Road, Shanghai 200072, P.R. China

E-mail: doctorlish77@gmail.com

*Contributed equally

Key words: neural stem cells, E-cadherin, inflammatory cytokines, spinal cord injury the peripheral nervous system, damaged neurons in central nervous system are rarely regenerated (2). Neural stem cells (NSCs), which are capable of generating multiple cell lineages, including neurons and glial cells, have the potential to repair and replace damaged neurons $(3,4)$. Up until now, stem cell-based repair strategies for SCI remain a highly investigated area of research. Various genetically modified stem cells have been transplanted into SCI models, although the results are not always successful $(5,6)$.

SCI is a complex injury that includes a primary injury (mechanical damage to the spinal cord) and a secondary injury (characterized by prolonged inflammatory cell infiltration, glial scar formation and tissue damage) (7). The altered microenvironment is no longer suitable for cell survival, and ultimately excessive glial scars hinder the regeneration of damaged axons $(8,9)$. A number of previous studies have confirmed that the transplantation of NSCs and embryonic stem cells (ESCs) promotes neurological recovery by decreasing the expression level of inflammatory cytokines $(10,11)$.

Cadherins, including $\mathrm{N}$-cadherin and E-cadherin, are a type of cell adhesion molecule that are vital for cell proliferation and migration (12). A previous study has demonstrated that $\mathrm{N}$-cadherin promotes recruitment and migration of NSCs (13). However, the expression of $\mathrm{N}$-cadherin and E-cadherin in NSCs are negatively correlated (14). Thus far, few studies have investigated the association between E-cadherin and NSCs.

In the present study, the expression of E-cadherin was observed in subcultured NSCs and differentiated NSCs. Furthermore, an E-cadherin overexpression lentivirus was constructed and transfected into NSCs in order to investigate the effect of E-cadherin on NSCs viability, migration, differentiation and neurosphere formation. An in vivo study was used to assess the survival rate of grafted NSCs-E-cadherin. Furthermore, by analyzing tissue repair, Basso Mouse Scale (BMS) scores and inflammatory cytokine expression levels the protective role of E-cadherin in SCI was assessed. Additionally, NSCs or NSCs-E-cadherin were co-cultured with mouse bone marrow-derived macrophages (BMDMs) in order to examine whether E-cadherin influenced the activation of macrophages.

\section{Materials and methods}

Cell culture and differentiation. Primary spinal cord derived NSCs were obtained from Animal Experimental Center of 
Tongji University (Shanghai, China) and cultured in Dulbecco's modified Eagle medium: Nutrient Mixture F-12 (DMEM/F12) supplemented with $1 \% \mathrm{~B} 27,20 \mathrm{ng} / \mathrm{ml}$ basic fibroblast growth factor (bFGF) and $20 \mathrm{ng} / \mathrm{ml}$ epidermal growth factor (EGF; all Thermo Fisher Scientific, Inc., Waltham, MA USA) at $37^{\circ} \mathrm{C}$ in an atmosphere containing $5 \% \mathrm{CO}_{2}$. Following 3-5 days of culture, the cells grew to form neurospheres and reached the highest density on the 7th day. Subsequently, a single cell suspension was prepared through the mechanical separation of the neurospheres, and the cells were subcultured every 3-5 days. Following the removal of bFGF and EGF, the cells were induced to differentiate using $1 \%$ fetal bovine serum (FBS; Gibco; Thermo Fisher Scientific, Inc.).

Construction of E-cadherin overexpression lentivirus. DNA was extracted from mouse NSCs. The primers were designed and synthesized with the following sequences: E-cadherin, forward 5'-GGGTCTTGCTATGTTGCC-3' and reverse 5'-GTTCCGCTCTGTCTTTGG-3', to amplify the E-cadherin sequence (hereafter known as fragment) using the PCR amplification kit (Omega Bio-tek, Inc., Norcross, GA, USA). The PCR thermocycling conditions were as follows: $94^{\circ} \mathrm{C}$ for $10 \mathrm{~min}$, followed by 30 cycles at $94^{\circ} \mathrm{C}$ for $30 \mathrm{sec}, 58^{\circ} \mathrm{C}$ for $30 \mathrm{sec}$ and $72^{\circ} \mathrm{C}$ for $90 \mathrm{sec}$, and a final extension of $94^{\circ} \mathrm{C}$ for $15 \mathrm{sec}, 60^{\circ} \mathrm{C}$ for $1 \mathrm{~min}, 94^{\circ} \mathrm{C}$ for $15 \mathrm{sec}$ and $60^{\circ} \mathrm{C}$ for $15 \mathrm{sec}$. The fragment was connected with the plasmid, PHY-027 (EF1A-MCS-CMV-zsGreen1-IRES-Puro; Shanghai Ruisai Biotechnology Co., Ltd, Shanghai, China), using EcoR I and $X b a$ I (Takara Biotechnology Co., Ltd., Dalian, China). Positive clones were identified using polymerase chain reaction and subsequently sequenced.

Two groups were established: The E-cadherin overexpression and control groups. The DNA plasmid mixture for the experimental group consisted of $1 \mu \mathrm{g} \mathrm{E}$-cadherin plasmid $(2 \mu \mathrm{g} / \mu \mathrm{l}), 0.75 \mu \mathrm{g}$ psPAX2 and $0.25 \mu \mathrm{g}$ pMD2.G (both Invitrogen; Thermo Fisher Scientific, Inc., Waltham, MA, USA) in OPTI-MEM (Promega Corporation, Madison, WI, USA). The DNA plasmid mixture for the control group consisted of $1 \mu \mathrm{g}$ empty plasmid $(1.8 \mu \mathrm{g} / \mu \mathrm{l}), 0.75 \mu \mathrm{g}$ psPAX2 and $0.25 \mu \mathrm{g}$ pMD2.G in OPTI-MEM in a total volume of $20 \mu \mathrm{l}$. A total of $6 \mu 1$ FuGENE $^{\circledR} 6$ (Promega Corporation) was added to OPTI-MEM, then this mixture was added to the DNA plasmid and incubated at room temperature for $20 \mathrm{~min}$. The mixture was then transferred into competent 293 T cells (Central Laboratory of Shanghai Tenth People's Hospital, Shanghai, China) for packaging and incubated at $37^{\circ} \mathrm{C}$ for $48 \mathrm{~h}$. Cell medium was then collected, and purified through ultrafiltration and ultracentrifugation. For each well, $150 \mu 1$ lentivirus $\left(2 \times 10^{8} \mathrm{TU} / \mathrm{ml}\right)$ with $5 \mu 1$ diluted polybrene in DMEM/F12 $(5 \mu \mathrm{g} / \mathrm{ml}$; Sigma-Aldrich; Merck KGaA, Darmstadt, Germany) was transfected into NSCs at room temperature and then the cells were incubated at $37^{\circ} \mathrm{C}$ for $48 \mathrm{~h}$ for subsequent experiments.

Viability and migration assays. Cell viability was evaluated using an MTT assay (Sigma-Aldrich; Merck KGaA). Cells were digested with trypsin, and $10 \%$ FBS was added to prevent serum interference. The cell concentration was adjusted to $4 \times 10^{4}$ cells/well. A total of $20 \mu \mathrm{l}$ MTT stock solution was added per well and incubated in $37^{\circ} \mathrm{C}$ for $4 \mathrm{~h}$. The samples were centrifuged and dimethyl sulfoxide was then added. Following $10 \mathrm{~min}$ of incubation, the absorbance values were recorded at $490 \mathrm{~nm}$ and the growth curve was drawn.

For migration, a Transwell assay was performed with a Transwell chamber (ECM550, Chemicon International, Inc., Billerica, MA, USA) was used. In the upper chamber, $1 \times 10^{5}$ NSCs in $100 \mu 1 \mathrm{DMEM} / \mathrm{F} 12$ and $0.05 \%$ bovine serum albumin (R\&D Systems, Inc., Minneapolis, MN, USA) were added. In the lower chamber, $300 \mu \mathrm{l}$ DMEM/F12 with 10\% FBS medium was added. Following $24 \mathrm{~h}$ incubation, the cells were stained with $1 \%$ crystal violet for $20 \mathrm{~min}$ at room temperature and counted under a light microscope (magnification, x100).

Western blotting. The protein samples were extracted using 1X SDS loading buffer. Protein concentration was determined using bicinchoninic acid assay quantification. A total of $20 \mu \mathrm{g}$ protein was loaded in each well, separated with SDS-PAGE (10\% gels) and blotted on polyvinylidene fluoride membranes (100 v for $120 \mathrm{~min}$ ). The membranes were blocked with Tris-buffered saline with Tween-20 (TBST) solution containing 5\% fat-free milk for $1 \mathrm{~h}$ at room temperature. Primary antibodies for E-cadherin (1:1,000; cat. no. MAB7481; R\&D Systems, Inc.) and $\beta$-actin (cat. no. ab8229; Abcam, Cambridge, UK) were incubated overnight at $4^{\circ} \mathrm{C}$. The membranes were washed using TBST 3 times and incubated with horseradish peroxidase-conjugated whole immunoglobulin G Affinity-Purified Antibodies (Goat Anti-Rabbit; 1:5,000; cat. no. 111-005-045; Jackson Immuno Research Laboratories, Inc., West Grove, PA, USA) at room temperature for $1 \mathrm{~h}$. Signals were detected using electrochemiluminescence reagent (Sigma-Aldrich; Merck $\mathrm{KGaA}$ ). The bands were analyzed using Image Lab software (version 3.0; Bio-Rad Laboratories, Inc., Hercules, CA, USA).

Reverse transcription-quantitative PCR (RT-qPCR). Total RNA was extracted from NSCs using TRIzol Reagent (Invitrogen; Thermo Fisher Scientific, Inc.). RNA quantification was performed using a NanoDrop ${ }^{\mathrm{TM}} 2000 \mathrm{c}$ Spectrophotometer (Thermo Fisher Scientific, Inc.). cDNA was synthesized using the Reverse Transcription kit (Applied Biosystems; Thermo Fisher Scientific, Inc.). qPCR was performed using SYBR-Green (Thermo Fisher Scientific, Inc.) and CFX384 Touch Real-Time (Bio-Rad Laboratories, Inc.). The PCR cycling conditions were as follows: $94^{\circ} \mathrm{C}$ for $10 \mathrm{~min}, 30$ cycles at $94^{\circ} \mathrm{C}$ for $30 \mathrm{sec}, 58^{\circ} \mathrm{C}$ for $30 \mathrm{sec}$ and $72^{\circ} \mathrm{C}$ for $1.5 \mathrm{~min}$, and finally $72^{\circ} \mathrm{C}$ for $5 \mathrm{~min}$. The primer sequences used were as follows: GAPDH, forward 5'-CCTGGAGAA ACCTGCCAAGTA-3' and reverse 5'-TCATACCAGGAA ATGAGCTTGAC-3'; interleukin (IL)-1 $\beta$, forward 5'-TGC CACCTTTTGACAGTGATG-3' and reverse 5'-TGTGCT GCTGCGAGATTTG-3'; IL-6, forward 5'-CAACCACGG CCTTCCCTACT-3' and reverse 5'-CACAACTCTTTTCTC ATTTCCACGA-3'; IL8, forward 5'-GCATCTCGGTGTAGA GCAAGG-3' and reverse 5'-CAGTGTCCCTATAGCCCA AGTTA-3'; monocyte chemoattractant protein 1 (MCP-1), forward 5'-CCTGGATCGGAACCAAATG-3' and reverse 5'-TGGTTGTGGAAAAGGTAGTGG-3'; inducible nitric oxide synthase (iNOS), forward 5'-TGTGCGAAGTGTCAG TGGCT-3' and reverse 5'-CCCTTTGTGCTGGGAGTCAT-3'; tumor necrosis factor $\alpha(\mathrm{TNF} \alpha)$, forward 5'-GAGTCCGGG CAGGTCTACTT-3' and reverse 5'-GTCACTGTCCCAGCA 
TCTTGT-3'; arginase-1 (Arg1), forward 5'-CTTTCCACG ACGGTGACACG-3' and reverse 5'-ACGCTAGGATCTGAT CGCGG-3'. GAPDH was used as an internal reference gene for normalization using the $2^{-\Delta \Delta \mathrm{Cq}}$ method (15).

SCI model. A total of 60 female, adult wild-type C57BL/6 mice (weighing $20 \mathrm{~g}$, aged 8-10 weeks old) were obtained from Animal Experimental Center of Tongji University (Shanghai, China) and used for injury model construction. The groups were as follows (15 mice/group): Sham, PBS treatment, NSCs treatment and E-cadherin overexpression NSCs (NSCs-E-cad) treatment. The mice were intraperitoneally anesthetized and a T9 laminectomy was performed to expose the spinal cord. Subsequently, the injury model was constructed by clamping the spinal cord at T9 for $30 \mathrm{sec}$ and the wound was stitched and disinfected. The mice were kept warm during the procedure. Following recovery from anesthesia, the mice displayed a lower limb motor deficit (motor function loss) and were housed in a temperature-controlled room at $27^{\circ} \mathrm{C}$. Food and water were provided ad libitum and the mice received manual bladder evacuation once a day following surgery.

The sham group did not undergo the SCI procedure. The NSCs and NSCs-E-cad group underwent the SCI procedure and subsequently had cell transplantations. The PBS group underwent the SCI procedure and were subsequently administered $2 \mu$ PBS 7 days after the procedure; PBS was injected at two sites (1-mm rostral and caudal to the injury epicenter).

Cell transplantation. Cell transplants were performed 7 days post injury (dpi). The mice were anesthetized and the laminectomy site was re-exposed. The NSCs and NSCs-E-cad were resuspended using PBS and the final concentration was adjusted to $1 \times 10^{5}$ cells $/ \mu 1$ (kept on ice prior to injection). For each mouse, $2-\mu 1$ cell suspensions were injected at two sites (1-mm rostral and caudal to the injury epicenter) using a 5- $\mu 1$ microinjector (Hamilton syringe; Hamilton Bonaduz AG, Bonaduz, Switzerland). The mice were injected at a rate of $0.5 \mu \mathrm{l} / \mathrm{min}$.

Immunohistochemistry. A total of 2 weeks after cell transplantation, animals were anesthetized, and perfused with PBS and $4 \%$ paraformaldehyde. The spinal cords were removed and post-fixed overnight at $4^{\circ} \mathrm{C}$. The fixed tissues were cryoprotected in $30 \%$ sucrose (Sigma-Aldrich; Merck KGaA) in PBS at $4^{\circ} \mathrm{C}$, and then embedded in optimal cutting temperature compound (Tissue Tek; Sakura Finetek USA, Inc., Torrance, CA, USA). Cryostat sections that were $10 \mu \mathrm{m}$ thick were cut and affixed to glass slides and stored at $-20^{\circ} \mathrm{C}$. Following this, the sections were processed for histochemistry, PBS with Tween 20 was used for permeabilization and 10\% FBS was used for blocking for $1 \mathrm{~h}$ at room temperature. Sections were then incubated with the primary antibodies for Nestin (cat. no. ab22035), neuron-specific class III $\beta$-tubulin (cat. no. ab18207) and glial fibrillary acidic protein (cat. no. ab7260; all 1:500; Abcam) at $4{ }^{\circ} \mathrm{C}$ overnight. Following this, goat anti-rabbit secondary antibodies (cat. no. ab150117; Abcam) were incubated for $1 \mathrm{~h}$ at room temperature. Subsequent to washing with PBS, nuclei were stained using 4',6-diamidino-2-phenylindole (Abcam) at room temperature for $30 \mathrm{~min}$. The processed slides were assessed under a fluorescent microscope (magnification, x200 or $\mathrm{x} 400)$.
Functional recovery assessment. Tissue sparing of the injured spinal cord was quantified using ImageJ 1.50 software (National Institutes of Health, Bethesda, MD, USA) on the basis of immunohistochemical stains. Motor function was assessed using BMS scores (9). The BMS score was recorded at the 1st day post-transplantation. A total of 3 days post-transplantation, $5 / 15$ mice in each group were sacrificed to obtain spinal cord tissue to investigate the differences in the expression of inflammatory cytokines among the three groups. A total of 6 weeks post transplantation, 7/10 in each group were sacrificed to assess cell survival and tissue sparing. The remaining mice were sacrificed at 3 months post-transplantation for checking cell survival in the long run.

Mouse BMDMpreparation. BMDMs were harvested from bone marrow, which was collected from the femoral shafts of 5 adult female C57BL/6 mice (Animal Experimental Center of Tongji University, Shanghai, China) aged 8 weeks old and weighing $20 \mathrm{~g}$ by flushing the marrow cavity with DMEM. Animals were housed in a room at a temperature of $24^{\circ} \mathrm{C}$ and $40 \%$ humidity with 12-h dark and light cycle; they had free access to standard food and water. Cells were cultured in DMEM supplemented with $10 \%$ FBS, $1 \%$ Penicillin-Streptomycin and $15 \%$ conditioned medium (with macrophage colony-stimulating factor; Gibco; Thermo Fisher Scientific, Inc.).

Interferon- $\gamma$ induction $(I N F-\gamma)$. BMDMs and NSCs were co-cultured in 6 -well plate $\left(5 \times 10^{5}\right.$ cells/well $)$ at $37^{\circ} \mathrm{C}$ in a $5 \%$ $\mathrm{CO}_{2}$ atmosphere for $12 \mathrm{~h}$. The cells were cultured in DMEM supplemented with 5\% newborn calf serum (Rocky Mountain Biologicals, Inc., Missoula, MT, USA). Then, treated with INF- $\gamma$ at a final concentration of $5 \mathrm{ng} / \mathrm{ml}$ for $6 \mathrm{~h}$. INF- $\gamma$ is usually used to induce the activation of M1 macrophages (16). The cells were centrifuged at 3,000 x g for $20 \mathrm{~min}$ at room temperature and the supernatant collected for subsequent experiments.

Statistical analysis. Images were quantified using ImageJ software with Java 1.8.0. Data are presented as the mean \pm standard deviation from three independent experiments. For MTT and BMS results, one-way analysis of variance (ANOVA) followed by the Student-Newman-Keuls (SNK) post hoc test for repeated measurements was used for statistical analysis. Non-normal distribution data was converted to normal distribution data by using logarithmic transformation performed by SPSS13.0 (SPSS, Inc., Chicago, IL, USA). A Student's t-test and one-way ANOVA followed by the SNK post hoc test were used for comparing the difference between two and more than two groups, respectively. Statistical analysis was performed with GraphPad Prism 7 software (GraphPad Software, Inc., La Jolla, CA, USA). P $<0.05$ was considered to indicate a statistically significant difference.

\section{Results}

NSCs form neurospheres and differentiate into neurons and astrocytes. NSCs were obtained from E13.5 of fetal mice spinal cord tissues and cultured in serum-free growth medium. The undifferentiated NSCs expressed the specific marker Nestin. Following 7 days in culture, NSCs were able to proliferate and 

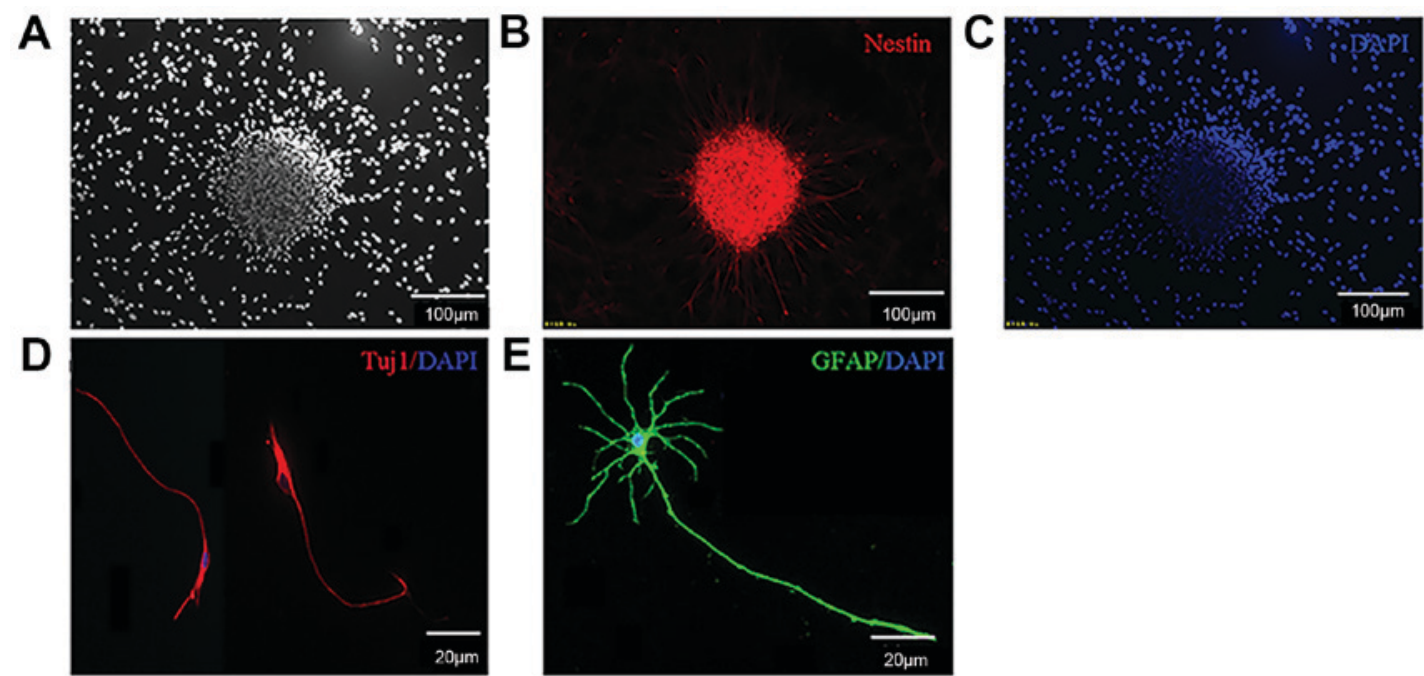

Figure 1. Isolated NSCs are able to proliferate and differentiate. (A) The undifferentiated NSCs were observed under microscopes. (B) NSCs could express Nestin and form neurospheres. (C) DAPI staining of NSCs. (D) NSCs were able to differentiate into neurons. (E) NSCs were able to differentiate into astrocytes. NSC, neural stem cells; DAPI, 4',6-diamidino-2-phenylindole; GFAP, glial fibrillary acidic protein; Tuji1, neuron-specific class III $\beta$-tubulin.
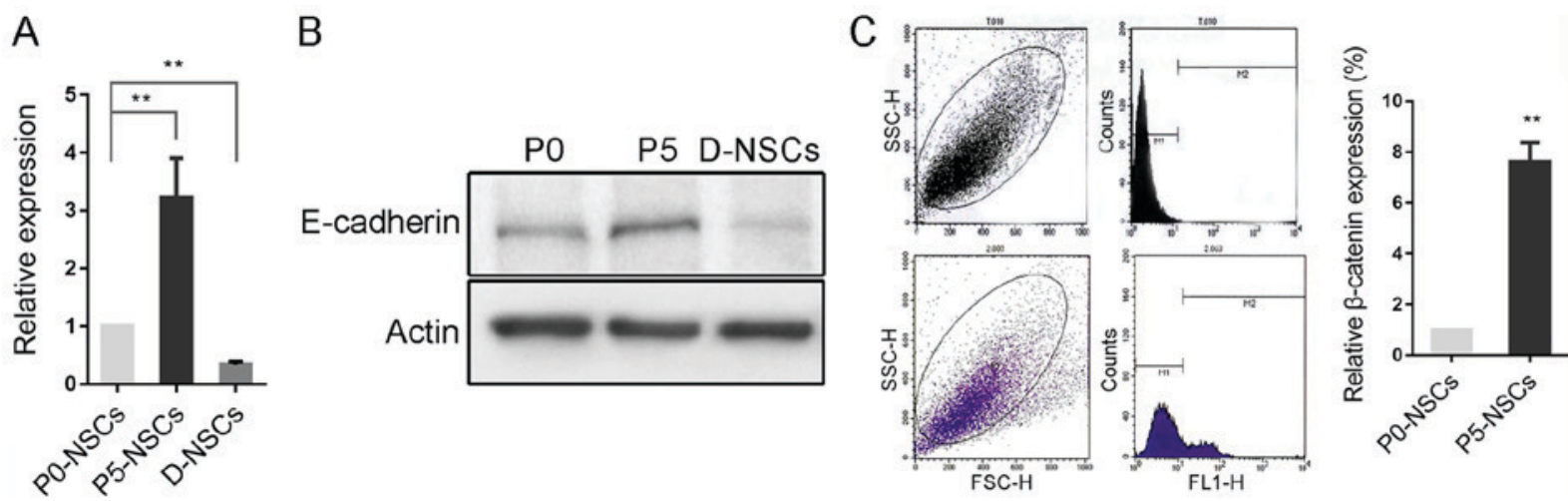

Figure 2. E-cadherin increases in subcultured NSCs but decreases subsequent to induced differentiation. (A) Reverse transcription-quantitative polymerase chain reaction revealed that E-cadherin was significantly increased in P5 NSCs compared with P0 cells, and decreased in differentiated NSCs. (B) The change was further confirmed by western blotting. (C) Expression of $\beta$-catenin, the core component of E-cadherin/ $\beta$-catenin complex, was increased in subcultured NSCs, as confirmed by flow cytometry. ${ }^{* *} \mathrm{P}<0.01$ as indicated or vs. P0-NSCs. NSC, neural stem cells; D-NSCs, differentiated NSCs; P0, primary cells; P5, passage 5.

form neurospheres (Fig. 1A-C). These primary neurospheres were mechanically separated into single cell suspensions and were further passaged every 2-3 days. A total of 3 days following the removal of bFGF and EGF, the cells differentiated into neurons and astrocytes (Fig. 1D and E).

E-cadherin expression increases in subcultured NSCs but decreases following induced differentiation. E-cadherin expression was significantly increased in passage 5 (P5) NSCs compared with P0 cells, and decreased following differentiation, which was further confirmed by western blotting ( $\mathrm{P}<0.01$; Fig. 2A and B). However, according to flow cytometry results, $\beta$-catenin, a key component of the E-cadherin/catenin complex (17) was also significantly increased in this process $(\mathrm{P}<0.01$; Fig. 2C).

Function of E-cadherin in regulating the biological behaviors of NSCs. To further investigate the function of E-cadherin in regulating the biological behaviors of NSCs, the lentivirus vector PHY-027 carrying E-cadherin and green fluorescent protein was constructed. As presented in Fig. 3A, the lentivirus was able to effectively promote E-cadherin protein expression following transfection into NSCs. The ectopic expression of E-cadherin appeared to significantly facilitate NSC viability, but significantly inhibited cell migration compared with the NSC group (all $\mathrm{P}<0.05$; Fig. $3 \mathrm{~B}$ and $\mathrm{C}$ ). Subsequent to induced differentiation, no significant difference in Tuj1-positive cells was identified between the two groups, indicating that E-cadherin did not significantly affect the fate determination of NSCs (Fig. 3D). The rate of neurosphere formation is considered a primary characteristic of stem cells (18). Notably, the rate of neurosphere formation was significantly higher in the E-cadherin group compared with the NC-NSC group, indicating that E-cadherin maintained the stemness of NSCs $(\mathrm{P}<0.01$; Fig. 3E).

E-cadherin improves the survival of grafted NSCs in the short term. A T9 laminectomy was performed to expose the spinal cord (Fig. 4A) The injury model was constructed by clamping the spinal cord at T9 for $30 \mathrm{sec}$, and two clear red 
A

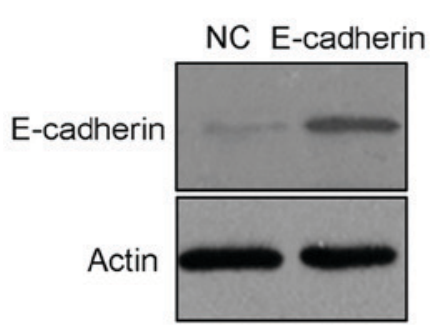

B

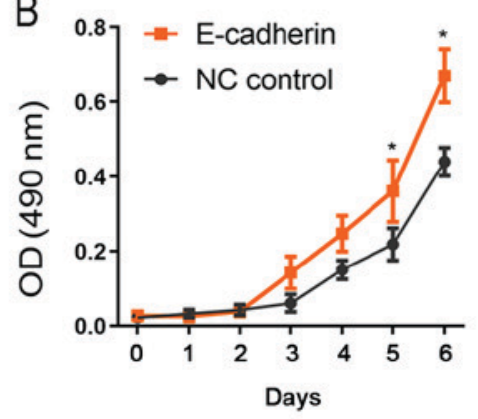

C

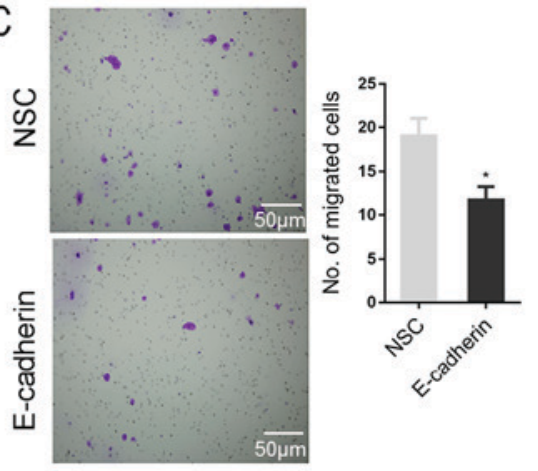

D

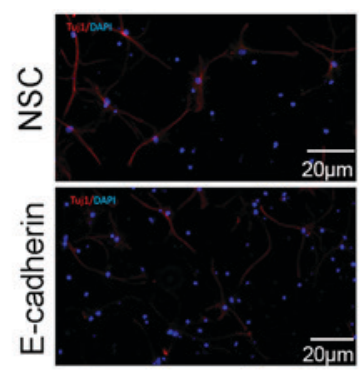

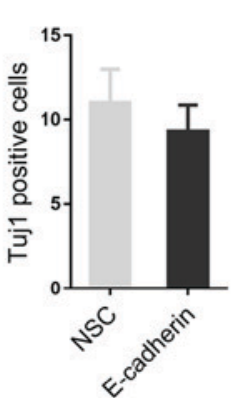

E

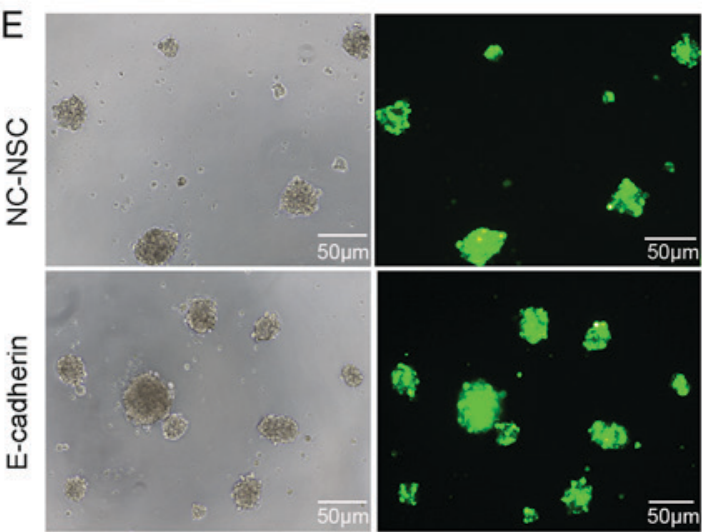

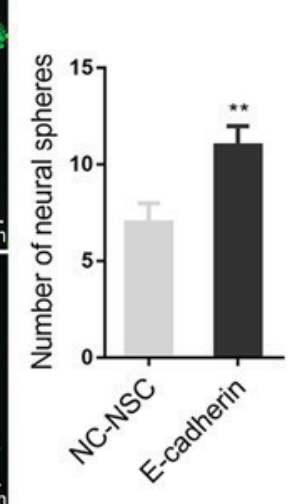

Figure 3. The role of E-cadherin in regulating the biological behaviors of NSCs. (A) Western blotting revealed that the lentivirus was able to significantly increase E-cadherin protein expression following transfection into NSCs. (B) MTT results indicated that E-cadherin appeared to facilitate NSCs viability. (C) Data obtained from a Transwell experiment demonstrated that E-cadherin inhibited cell migration. (D) The immunostaining revealed that E-cadherin did not affect the fate determination of NSCs, as it did not alter the percentage of differentiated neurons (Tuj1). (E) E-cadherin increased the number of neural spheres and maintained the stemness of NSCs. ${ }^{*}<<0.05$ and ${ }^{* *} \mathrm{P}<0.01$ vs. NSC. NSC, neural stem cells; Tuj1, neuron-specific class III $\beta$-tubulin; NC, negative control; DAPI, 4',6-diamidino-2-phenylindole.

lines were observed at either side of the midline (Fig. 4B). Subsequent to surgery, the mice displayed a lower limb motor deficit. Disruption of tissue was revealed in the injured spinal cord (Fig. 4C). A total of 3 months post-surgery, the injured spinal cord was able to form a large cavity surrounded by GFAP-positive glial cells (Fig. 4D). The grafted NSCs were able to migrate to the lesion site and increased cell survival around the epicenter was observed in the E-cadherin group at 14 dpi compared with the control group ( $\mathrm{P}<0.05$; Fig. 4E).

E-cadherin served as a 'neuroprotector' following SCI. A total of 6 weeks post-transplantation, mice tissues were sectioned and stained with GFAP. As presented in Fig. 5A, compared with the sham group, PBS treatment resulted in large tissue cavities, and the caudal and rostral of the lesion site were not fully connected. Furthermore, the total volume of the spinal cord was substantially reduced. Conversely, the E-cadherin group demonstrated increased tissue sparing compared with the PBS and NSCs groups, with the exception of $-2,000$ and $-3,000 \mathrm{um}$; at these distances, tissue sparing in the E-cadherin group was significantly increased compared with the PBS group (all $\mathrm{P}<0.05$; Fig. 5B). In line with the aforementioned results, the highest BMS scores were obtained in the E-cadherin group. BMS scores in the E-cadherin group were significantly greater compared with the PBS and NSC groups 3-6 weeks after transplantation, but only significantly greater compared with the PBS group 2 weeks after transplantation (all $\mathrm{P}<0.05$; Fig. 5C). These results indicate improved motor functional recovery in the E-cadherin group compared with the PBS and NSC groups. Therefore, suggesting that grafted NSCs with highly expressed E-cadherin may promote motor function recovery and create an improved microenvironment for neural regeneration.

E-cadherin alleviates secondary damage by reducing the release of inflammatory cytokines. To determine whether E-cadherin was able to promote motor function recovery via regulating inflammatory responses following SCI, several associated pro-inflammatory cytokines, including IL-1 $\beta$, IL-6, IL-8, MCP-1, iNOS and TNF- $\alpha$ were detected in the injured spinal cord tissue in each group 3 days post-transplantation. These cytokines were significantly higher in the injured spinal cord group compared with the sham group, suggesting that the inflammatory response was activated following SCI (all $\mathrm{P}<0.01$; Fig. 6A). The release of these cytokines was significantly reduced in the E-cadherin group compared with the NSC group (all $\mathrm{P}<0.05$ ). Notably, iNOS and TNF- $\alpha$ are regarded as M1 macrophage markers, and M1 macrophage infiltration is considered to be the primary cause of further damage by secreting inflammatory cytokines following primary mechanical injury (16). On these grounds, mouse BMDMs were cultured with NSCs or NSCs-E-cadherin to investigate whether E-cadherin influenced M1 macrophage activation. Upon type II IFN- $\gamma$ 
A

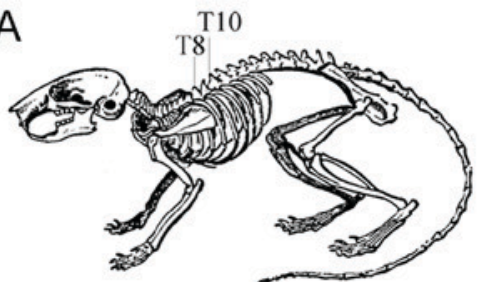

D

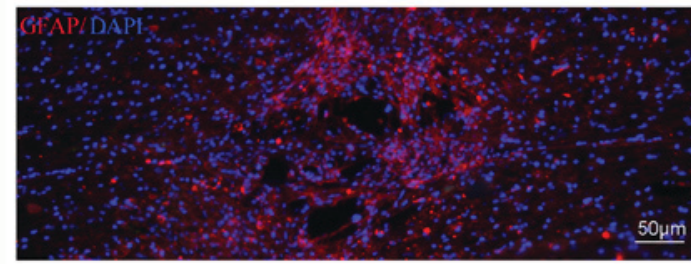

B

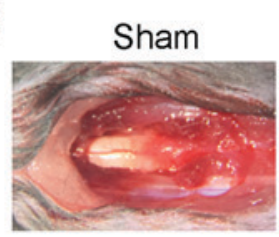

$\mathrm{SCl}$

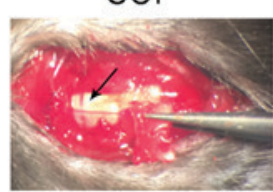

C

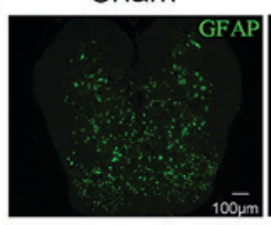

$\mathrm{SCl}$

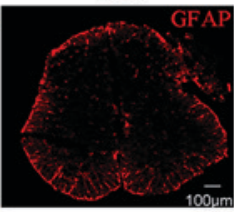

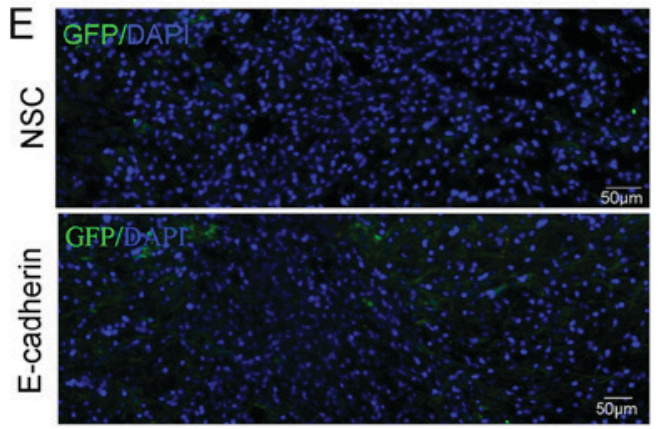

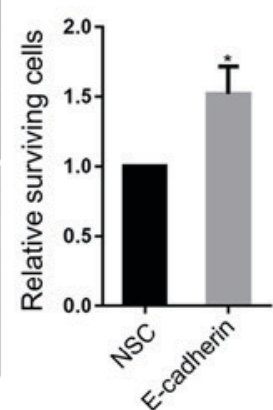

Figure 4. E-cadherin improved the survival of grafted NSCs in the short term. (A) T9 laminectomy was performed to exposure spinal cord. (B) Subsequent to clamping the spinal cord at $\mathrm{T} 9$ for $30 \mathrm{sec}$, two red lines were observed at either sides of the midline (black arrow). (C) The disruption of the tissue was observed in the injured spinal cord. (D) A total of 3 months post surgery, the injured spinal cord was able to form a large cavity surrounded by GFAP positive glial cells (E) Transplanted NSCs were able to migrate to the lesion site, and E-cadherin improved the survival of NSCs around the epicenter in the short term. ${ }^{*} \mathrm{P}<0.05$ vs. NSC. NSC, neural stem cells; GFAP, glial fibrillary acidic protein. DAPI, 4',6-diamidino-2-phenylindole; GFAP, glial fibrillary acidic protein; GFP, green fluorescent protein; SCI, spinal cord injury.

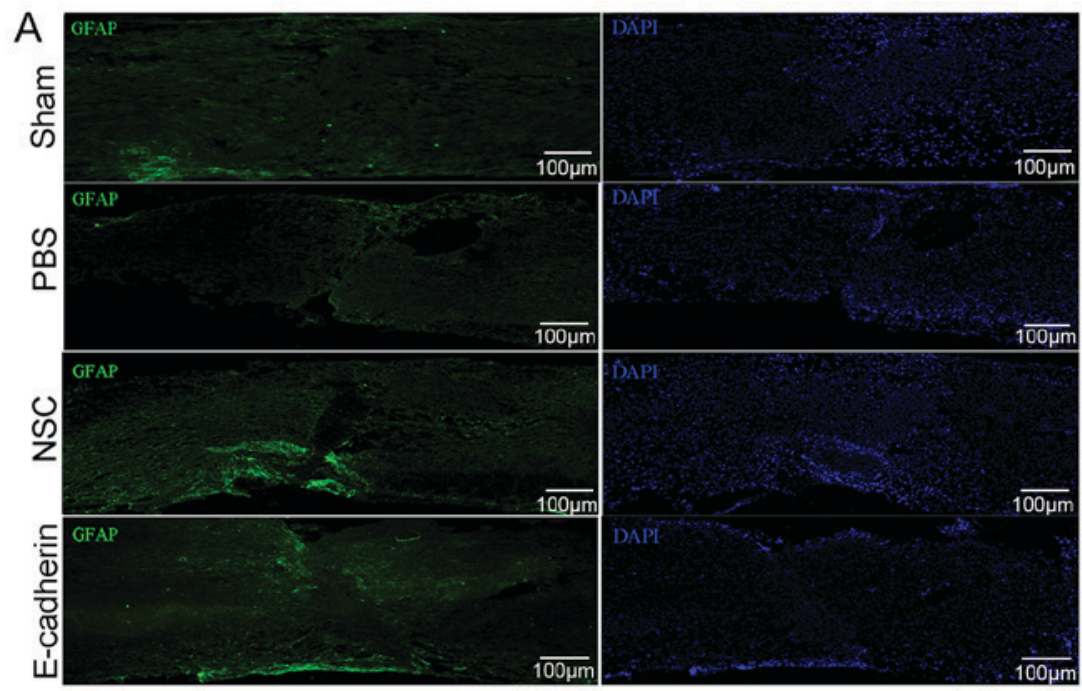

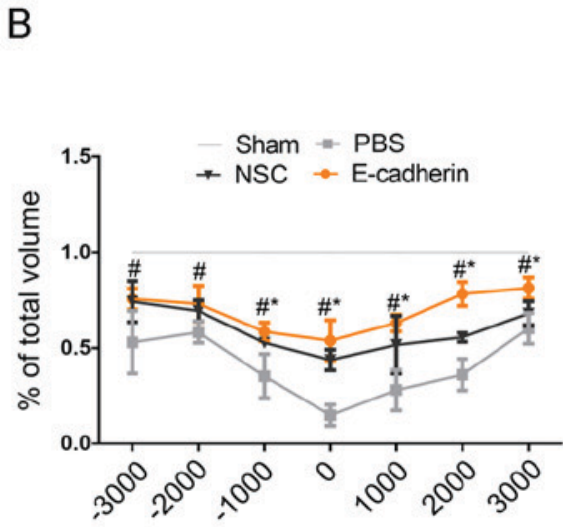

Distance from epicenter $(\mu \mathrm{m})$

C

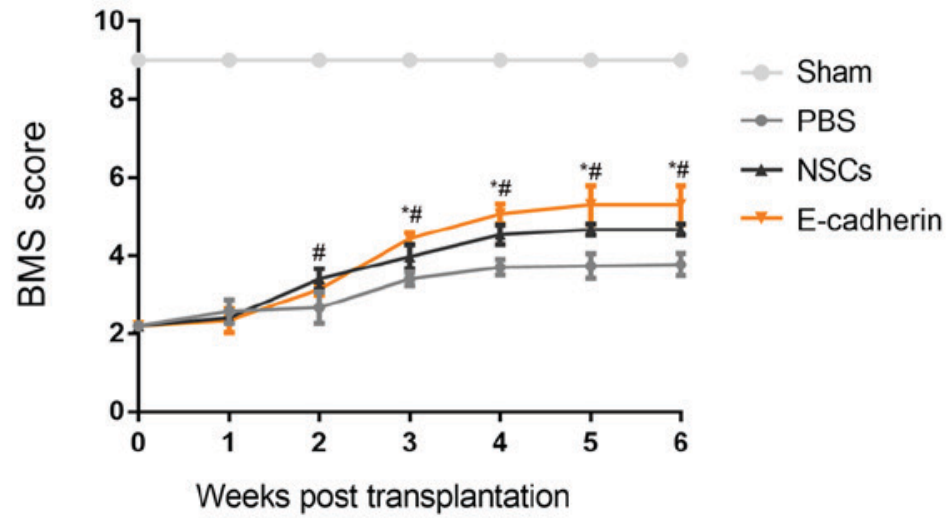

Figure 5. Grafted NSCs with highly expressed E-cadherin are able to promote motor function recovery and create a better microenvironment for neural regeneration. (A) Immunostaining of GFAP in spinal cord tissues. (B) NSCs with E-cadherin group increased tissue sparing the most compared with PBS treatment and NSCs transplantation. (C) NSCs with E-cadherin transplantation obtained the highest BMS scores. "P<0.05 vs. NSCs; ${ }^{*} \mathrm{P}<0.05$ vs. PBS. NSCs, neural stem cells; BMS, Basso Mouse Scale; GFAP, glial fibrillary acidic protein; DAPI, 4',6-diamidino-2-phenylindole. 

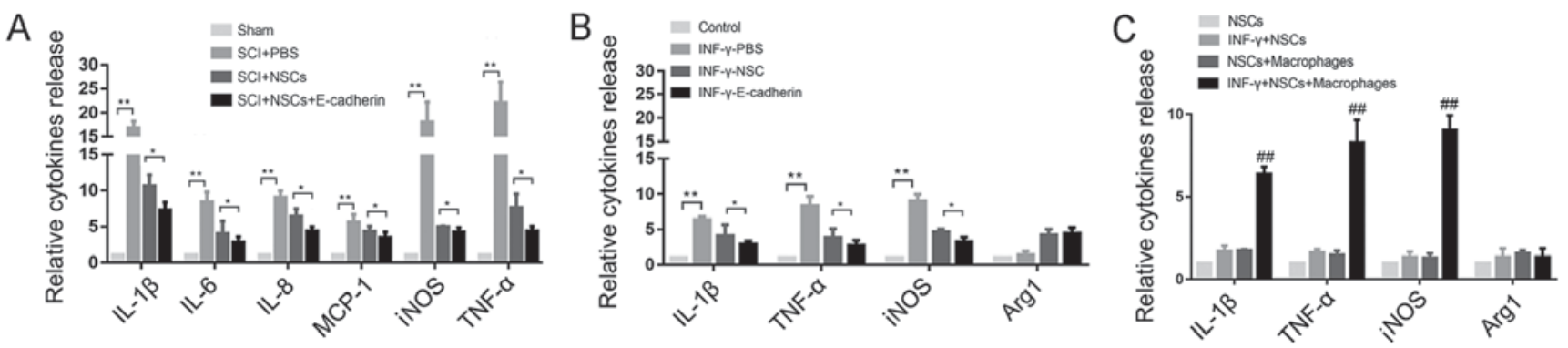

3 days after cell transplantation

Figure 6. E-cadherin alleviated secondary damage by reducing the release of inflammatory cytokines. (A) The release of inflammatory cytokines (IL-1 $\beta$, IL-6, IL-8, MCP-1, iNOS and TNF- $\alpha$ ) was increased in the PBS group 3 days after transplantation. (B) INF- $\gamma$ was able to induce the ectopic expression of IL-1 $\beta$, iNOS and TNF- $\alpha$ released by activated macrophages, and NSCs alone were able to reduce the expression of these cytokines, while NSCs with E-cadherin exhibited the most marked inhibitory effect. (C) Cytokines were detected in NSCs alone in response to INF- $\gamma$ treatment. No obvious difference was observed before and after INF- $\gamma$ treatment. ${ }^{*} \mathrm{P}<0.05,{ }^{* *} \mathrm{P}<0.01$ as indicated; ${ }^{\# \#} \mathrm{P}<0.01$ vs. NSCs. NSCs, neural stem cells; IL, interleukin; MCP-1, monocyte chemoattractant protein 1; iNOS, inducible nitric oxide synthase; TNF- $\alpha$, tumor necrosis factor $\alpha$; IFN- $\gamma$, type II interferon.

induction, the expression of IL-1 $\beta$, iNOS and TNF- $\alpha$ in the PBS group was significantly increased compared with the control group (all $\mathrm{P}<0.01$; Fig. $6 \mathrm{~B}$ ), indicating that the M1 macrophage was activated. Compared with the NSC group, E-cadherin exhibited the most significant inhibitory effect on the release of these cytokines (all $\mathrm{P}<0.05$ ). To rule out the possibility that the pro-cytokines were partly released by NSCs, cyrtokine release was assessed in the NSC in response to INF- $\gamma$ treatment. The release of cytokines was only significantly increased, compared with the NSCs, in NSC and macrophage co-cultures (Fig. 6C). No significant difference was observed prior to and post-INF- $\gamma$ treatment in NSCs alone, indicating that NSCs may respond to INF- $\gamma$, but not release inflammatory cytokines. These results may partly explain why E-cadherin promoted motor function recovery.

\section{Discussion}

The traditional concept that injured neurons cannot regenerate has been challenged since NSCs were identified in 1992 (19). The hallmark characteristics of NSCs include the ability to self-renew and generate multiple cell lineages, including neurons, astrocytes and oligodendrocytes (20). Consequently, grafted NSCs have the potential to replace injured neurons and reconstruct neural circuits in neurodegenerative/traumatic diseases (21). Basic transplantation research including clinical applications have been performed for decades, but despite the few favorable results gained, there are still numerous unsolved issues, including a lower grafted cell survival rate, restricted differentiation of glial lineage and the cells rarely integrating with host cells (22-24).

In addition to neuronal differentiation advantages, the anti-inflammatory effects of NSCs have been demonstrated in vitro and in vivo following SCI. NSCs have been demonstrated to influence the phenotypes of macrophages and secrete immunosuppressive factors (25-28). The pathophysiology of acute SCI comprises the primary injury (mechanical damage of the spinal cord) and the secondary injury (a cascade process initiated hours to days after the primary injury) that is characterized by a prolonged inflammatory response resulting in inflammatory cell infiltration, glial scar formation and further tissue damage (29-32).
The adhesion protein E-cadherin has been identified as an epithelial-to-mesenchymal transition marker and is vital for tumor progression and metastasis $(33,34)$. As a transmembrane protein, its functions involve the regulation of cell adhesion and mediation of extracellular matrix signaling (35). E-cadherin has been recognized as one of the key factors to maintain the morphological structure and functional integrity of epithelial cells (36-38). Several studies have confirmed the function of E-cadherin in stem cells. For example, Eastham et al (39) identified E-cadherin as a marker for undifferentiated human ESCs (hESCs), which were used to demarcate differentiated and undifferentiated hESCs. D'Amour et al (40) demonstrated that E-cadherin is co-expressed with typical undifferentiated markers of hESCs (including stage-specific embryonic antigen-4) and its expression is decreased immediately following the induction of differentiation. Furthermore, E-cadherin directly regulates hESC survival and self-renewal. Upregulated E-cadherin substantially enhances the cloning efficiency and pluripotency of hESCs (41).

However, the function of E-cadherin in regulating the biological behavior of spinal cord-derived NSCs remains unknown. In the present study, the increased expression of E-cadherin in subcultured NSCs (P5) compared with P0 cells was observed. $\beta$-catenin, localized at the core of the E-cadherin/catenin complex, was also increased. Notably, a previous study revealed that $\beta$-catenin was involved in Wnt signaling, which has been proven to control mouse embryonic stem cell stemness (42). MTT and Transwell assays were performed, and the results revealed that the ectopic expression of E-cadherin was able to maintain the stemness of NSCs by enhancing cell viability and abrogating cell motility. Similar results have been obtained in other cell types (43-45). Jin et al (46) demonstrated that germline stem cells (GSCs) expressing more E-cadherin become more competitive compared with the neighboring GSCs occupying the niche, which is the fundamental requirement for cell self-renewal. Furthermore, E-cadherin-mediated cell adhesion has been confirmed to serve the principal role to preserve the stemness state of mouse ESCs (47). Karpowicz et al (48) proposed that E-cadherin regulates the self-renewal of embryonic forebrain ventricles derived NSCs. 
Since E-cadherin had a positive effect on NSC viability in vitro, NSCs-E-cadherin were transplanted into the SCI mice to further investigated whether it promoted long-term cell survival in vivo. The grafted cells were revealed to be capable of surviving. Furthermore, they were localized around damaged axons. Unexpectedly, E-cadherin demonstrated no significant effect on cell survival in the long term, and the altered niche was favorable to glial differentiation. However, NSCs with highly expressed E-cadherin substantially maximized spinal cord integrity and ultimately improved motor function recovery. Those combined results indicated that E-cadherin served a neuroprotective role in SCI.

The expression levels of inflammatory cytokines, including IL-1 $\beta$, IL-6, IL-8, MCP-1, iNOS and TNF- $\alpha$, were recorded. By comparison, E-cadherin substantially reduced the expression of these cytokines and demonstrated the highest inhibition compared with the PBS and NSCs groups. This implies that E-cadherin relieves the inflammatory response following SCI. Interestingly, as iNOS and TNF- $\alpha$ are regarded as M1 macrophage markers and M1 macrophage infiltration is considered to be the main cause of secondary injury cascade (14), the present study co-cultured BMDMs with NSCs or NSCs-E-cadherin to investigate whether E-cadherin influenced M1 macrophage activation. As expected, compared with the PBS and NSC groups, E-cadherin exhibited the most significant inhibitory effect on IL-1 $\beta$, iNOS and TNF- $\alpha$ expression induced by IFN- $\gamma$. These results partly explained why E-cadherin promoted motor functional recovery following SCI. At present, there are few studies that have investigated the association between E-cadherin and inflammation. Shie et al (49) reported lower E-cadherin expression in patients with interstitial cystitis, which is associated with higher apoptotic cell numbers, higher visual analog scale pain scores and chronic inflammation. The adherens junction complex (E-cadherin/ $\beta$-catenin), together with tight junctions and desmosomes, controls the epithelial cell-to-cell adherence and forms a selective barrier. Disruption of this barrier is associated with inflammatory bowel disease (50). It has been suggested that E-cadherin mediated cell-to-cell connection, which is vital for the maintenance of epithelial integrity, the disruption of which is sufficient to initiate the development of inflammation (51-53). Tsai et al (54) revealed that proinflammatory cytokines and activated immune cells contribute to the disarrangement of the E-cadherin/catenin complex and cause the shedding of E-cadherin from the cell surface, and substantially elevated E-cadherin in plasma was identified in patients with pelvic inflammatory disease. In addition, activated neutrophils have been confirmed to induce the decomposition of the E-cadherin/ $\beta$-catenin complex, resulting in the enhanced migration of immune cells (55). Therefore, providing an improved cell microenvironment by relieving the inflammatory response subsequent to SCI, along with cell transplantation or endogenous NSCs activation, may be a promising strategy for treating SCI. In conclusion, E-cadherin may maintain NSCs in an undifferentiated state by promoting cell viability and neurosphere formation. Grafted NSCs with highly expressed E-cadherin facilitated motor functional recovery following SCI by reducing the release of inflammatory cytokines and provide a favorable microenvironment for neural regeneration.

\section{Acknowledgements}

Not applicable.

\section{Funding}

No funding was received.

\section{Availability of data and materials}

The datasets used and/or analyzed during the current study are available from the corresponding author on reasonable request.

\section{Authors' contributions}

DC and SL planned the study. DC wrote the manuscript and performed the primary experiments. SH and JL were responsible for the construction of SCI models, provided general coordination of the study and revised the manuscript. All authors read and approved the final manuscript.

\section{Ethics approval and consent to participate}

All applicable international, national and institutional guidelines for the care and use of animals were followed. Ethical approval for animal (mice) use was provided by the Ethics Committee of Shanghai Tenth People's Hospital, Tongji University School of Medicine (Shanghai, China).

\section{Patient consent to participate}

Not applicable.

\section{Competing interests}

The authors declare that they have no competing interests.

\section{References}

1. Gensel JC, Donnelly DJ and Popovich PG: Spinal cord injury therapies in humans: An overview of current clinical trials and their potential effects on intrinsic CNS macrophages. Expert Opin Ther Targets 15: 505-518, 2011.

2. Tavares I: Human neural stem cell transplantation in spinal cord injury models: How far from clinical application? Stem Cell Res Ther 4: 61, 2013.

3. Lu Y and Wang MY: Neural stem cell grafts for complete spinal cord injury. Neurosurgery 71: N13-N15, 2012.

4. Pomeshchik Y, Puttonen KA, Kidin I, Ruponen M, Lehtonen S, Malm T, Åkesson E, Hovatta O and Koistinaho J: Transplanted human induced pluripotent stem cell-derived neural progenitor cells do not promote functional recovery of pharmacologically immunosuppressed mice with contusion spinal cord injury. Cell Transplant 24: 1799-1812, 2015.

5. Salewski RP, Mitchell RA, Li L, Shen C, Milekovskaia M, Nagy A and Fehlings MG: Transplantation of induced pluripotent stem cell-derived neural stem cells mediate functional recovery following thoracic spinal cord injury through remyelination of axons. Stem Cells Transl Med 4: 743-754, 2015.

6. Sugai K, Nishimura S, Kato-Negishi M, Onoe H, Iwanaga S, Toyama Y, Matsumoto M, Takeuchi S, Okano $\mathrm{H}$ and Nakamura M: Neural stem/progenitor cell-laden microfibers promote transplant survival in a mouse transected spinal cord injury model. J Neurosci Res 93: 1826-1838, 2015.

7. Gonzalez R, Glaser J, Liu MT, Lane TE and Keirstead HS: Reducing inflammation decreases secondary degeneration and functional deficit after spinal cord injury. Exp Neurol 184: 456-463, 2003. 
8. Conti A, Cardali S, Genovese T, Di Paola R and La Rosa G: Role of inflammation in the secondary injury following experimental spinal cord trauma. J Neurosurg Sci 47: 89-94, 2003.

9. Machova Urdzikova L, Karova K, Ruzicka J, Kloudova A, Shannon C, Dubisova J, Murali R, Kubinova S, Sykova E, Jhanwar-Uniyal $\mathrm{M}$ and Jendelova P: The anti-inflammatory compound curcumin enhances locomotor and sensory recovery after spinal cord injury in rats by immunomodulation. Int $\mathrm{J}$ Mol Sci 17: pii: E49, 2015.

10. Han D, Wu C, Xiong Q, Zhou L and Tian Y: Anti-inflammatory mechanism of bone marrow mesenchymal stem cell transplantation in rat model of spinal cord injury. Cell Biochem Biophys 71: 1341-1347, 2015.

11. Karavelioglu E, Gönül Y, Kokulu S, Hazman Ö, Bozkurt F, Koçak A and Eser O: Anti-inflammatory and antiapoptotic effect of interleukine-18 binding protein on the spinal cord ischemia-reperfusion injury. Inflammation 37: 917-923, 2014.

12. Gao J, Grill RJ, Dunn TJ, Bedi S, Labastida JA, Hetz RA, Xue H, Thonhoff JR, DeWitt DS, Prough DS, et al: Human neural stem cell transplantation-mediated alteration of microglial/macrophage phenotypes after traumatic brain injury. Cell Transplant 25: 1863-1877, 2016.

13. Pellegatta S, Tunici P, Poliani PL, Dolcetta D, Cajola L, Colombelli C, Ciusani E, Di Donato S and Finocchiaro G: The therapeutic potential of neural stem/progenitor cells in murine globoid cell leukodystrophy is conditioned by macrophage/microglia activation. Neurobiol Dis 21: 314-323, 2006.

14. Klingener M, Chavali M, Singh J, McMillan N, Coomes A, Dempsey PJ, Chen EI and Aguirre A: N-cadherin promotes recruitment and migration of neural progenitor cells from the SVZ neural stem cell niche into demyelinated lesions. J Neurosci 34: 9590-9606, 2014.

15. Livak KJ and Schmittgen TD: Analysis of relative gene expression data using real-time quantitative PCR and the 2(-Delta Delta C(T)) method. Methods 25: 402-408, 2001

16. Karin $M$, Lawrence $T$ and Nizet V: Innate immunity gone awry: Linking microbial infections to chronic inflammation and cancer. Cell 124: 823-835, 2006.

17. Nakamura E, Sugihara H, Bamba $M$ and Hattori T: Dynamic alteration of the E-cadherin/catenin complex during cell differentiation and invasion of undifferentiated-type gastric carcinomas. J Pathol 205: 349-358, 2005.

18. Ma ZZ, Fan L, Huang JL and Pan XJ: A novel method to derive and expand mice neural stem cells efficiently without neuro-sphere formation. Int J Clin Exp Med 8: 12834-12841, 2015.

19. Reynolds BA, Tetzlaff W and Weiss S: A multipotent EGF-responsive striatal embryonic progenitor cell produces neurons and astrocytes. J Neurosci 12: 4565-4574, 1992.

20. Anderson DJ: Stem cells and pattern formation in the nervous system: The possible versus the actual. Neuron 30: 19-35, 2001.

21. Bacigaluppi M, Pluchino S, Peruzzotti-Jametti L, Kilic E, Kilic U, Salani G, Brambilla E, West MJ, Comi G, Martino G and Hermann DM: Delayed post-ischaemic neuroprotection following systemic neural stem cell transplantation involves multiple mechanisms. Brain 132: 2239-2251, 2009.

22. Robert AA,Zamzami M, Sam AE, Al Jadid M and Al Mubarak S: The efficacy of antioxidants in functional recovery of spinal cord injured rats: An experimental study. Neurol Sci 33: 785-791, 2012.

23. Ravikumar R, Narayanan S, Baskar S, Senthil Nagarajan R and Abraham S: Autologous stem cell injection for spinal cord injury-a clinical study from India. J Stem Cells Regen Med 3: 24-25, 2007.

24. Seebach C, Henrich D, Meier S, Nau C, Bonig H and Marzi I: Safety and feasibility of cell-based therapy of autologous bone marrow-derived mononuclear cells in plate-stabilized proximal humeral fractures in humans. J Transl Med 14: 314, 2016.

25. Kumar AA, Kumar SR, Narayanan R, Arul K and Baskaran M: Autologous bone marrow derived mononuclear cell therapy for spinal cord injury: A phase I/II clinical safety and primary efficacy data. Exp Clin Transplant 7: 241-248, 2009.

26. Einstein O, Karussis D, Grigoriadis N, Mizrachi-Kol R, Reinhartz E, Abramsky O and Ben-Hur T: Intraventricular transplantation of neural precursor cell spheres attenuates acute experimental allergic encephalomyelitis. Mol Cell Neurosci 24: 1074-1082, 2003.

27. Pluchino S, Zanotti L, Rossi B, Brambilla E, Ottoboni L, Salani G, Martinello M, Cattalini A, Bergami A, Furlan R, et al: Neurosphere-derived multipotent precursors promote neuroprotection by an immunomodulatory mechanism. Nature 436: 266-271, 2005
28. Aharonowiz M, Einstein O, Fainstein N, Lassmann $\mathrm{H}$, Reubinoff B and Ben-Hur T: Neuroprotective effect of transplanted human embryonic stem cell-derived neural precursors in an animal model of multiple sclerosis. PLoS One 3: e3145, 2008.

29. Cheng Z, Zhu W, Cao K, Wu F, Li J, Wang G, Li H, Lu M, Ren Y and $\mathrm{He} \mathrm{X}$ : Anti-inflammatory mechanism of neural stem cell transplantation in spinal cord injury. Int J Mol Sci 17: pii: E1380, 2016.

30. Mautes AE, Weinzierl MR, Donovan F and Noble LJ: Vascular events after spinal cord injury: Contribution to secondary pathogenesis. Phys Ther 80: 673-687, 2000.

31. Fleming JC, Norenberg MD, Ramsay DA, Dekaban GA, Marcillo AE, Saenz AD, Pasquale-Styles M, Dietrich WD and Weaver LC: The cellular inflammatory response in human spinal cords after injury. Brain 129: 3249-3269, 2006.

32. Hausmann ON: Post-traumatic inflammation following spinal cord injury. Spinal Cord 41: 369-378, 2003.

33. Liu Y, Ye H, Satkunendrarajah K, Yao GS, Bayon Y and Fehlings MG: A self-assembling peptide reduces glial scarring, attenuates post-traumatic inflammation and promotes neurological recovery following spinal cord injury. Acta Biomater 9: 8075-8088, 2013

34. van Roy F: Beyond E-cadherin: Roles of other cadherin superfamily members in cancer. Nat Rev Cancer 14: 121-134, 2014

35. Onder TT, Gupta PB, Mani SA, Yang J, Lander ES and Weinberg RA: Loss of E-cadherin promotes metastasis via multiple downstream transcriptional pathways. Cancer Res 68: 3645-3654, 2008

36. Vleminckx K, Vakaet L Jr, Mareel M, Fiers W and van Roy F: Genetic manipulation of E-cadherin expression by epithelial tumor cells reveals an invasion suppressor role. Cell 66: 107-119, 1991.

37. Christofori G and Semb H: The role of the cell-adhesion molecule E-cadherin as a tumour-suppressor gene. Trends Biochem Sci 24: 73-76, 1999.

38. Frixen UH, Behrens J, Sachs M, Eberle G, Voss B, Warda A, Löchner D and Birchmeier W: E-cadherin-mediated cell-cell adhesion prevents invasiveness of human carcinoma cells. J Cell Biol 113: 173-185, 1991

39. Eastham AM, Spencer H, Soncin F, Ritson S, Merry CL, Stern PL and Ward CM: Epithelial-mesenchymal transition events during human embryonic stem cell differentiation. Cancer Res 67: 11254-11262, 2007.

40. D'Amour KA, Agulnick AD, Eliazer S, Kelly OG, Kroon E and Baetge EE: Efficient differentiation of human embryonic stem cells to definitive endoderm. Nat Biotechnol 23: 1534-1541, 2005.

41. Xu Y, Zhu X, Hahm HS, Wei W, Hao E, Hayek A and Ding S: Revealing a core signaling regulatory mechanism for pluripotent stem cell survival and self-renewal by small molecules. Proc Natl Acad Sci USA 107: 8129-8134, 2010.

42. Li L, Wang BH, Wang S, Moalim-Nour L, Mohib K, Lohnes D and Wang L: Individual cell movement, asymmetric colony expansion, rho-associated kinase, and E-cadherin impact the clonogenicity of human embryonic stem cells. Biophys J 98: 2442-2451, 2010

43. Hao J, Li TG, Qi X, Zhao DF and Zhao GQ: WNT/beta-catenin pathway up-regulates Stat3 and converges on LIF to prevent differentiation of mouse embryonic stem cells. Dev Biol 290: 81-91, 2006

44. Hawkins K, Mohamet L, Ritson S, Merry CL and Ward CM: E-cadherin and, in its absence, $\mathrm{N}$-cadherin promotes Nanog expression in mouse embryonic stem cells via STAT3 phosphorylation. Stem Cells 30: 1842-1851, 2012.

45. Maeda K, Takemura M, Umemori M and Adachi-Yamada T: E-cadherin prolongs the moment for interaction between intestinal stem cell and its progenitor cell to ensure Notch signaling in adult Drosophila midgut. Genes Cells 13: 1219-1227, 2008.

46. Jin Z, Kirilly D, Weng C, Kawase E, Song X, Smith S, Schwartz J and Xie T: Differentiation-defective stem cells outcompete normal stem cells for niche occupancy in the Drosophila ovary. Cell Stem Cell 2: 39-49, 2008.

47. del Valle I, Rudloff S, Carles A, Li Y, Liszewska E, Vogt R and Kemler R: E-cadherin is required for the proper activation of the Lifr/Gp130 signaling pathway in mouse embryonic stem cells. Development 140: 1684-1692, 2013.

48. Karpowicz P, Willaime-Morawek S, Balenci L, DeVeale B, Inoue $\mathrm{T}$ and van der Kooy D: E-Cadherin regulates neural stem cell self-renewal. J Neurosci 29: 3885-3896, 2009. 
49. Shie JH and Kuo HC: Higher levels of cell apoptosis and abnormal E-cadherin expression in the urothelium are associated with inflammation in patients with interstitial cystitis/painful bladder syndrome. BJU Int 108: E136-E141, 2011.

50. Mehta S, Nijhuis A, Kumagai T, Lindsay J and Silver A: Defects in the adherens junction complex (E-cadherin/ $\beta$-catenin) in inflammatory bowel disease. Cell Tissue Res 360: 749-760, 2015.

51. Zbar AP, Simopoulos C and Karayiannakis AJ: Cadherins: An integral role in inflammatory bowel disease and mucosal restitution. J Gastroenterol 39: 413-421, 2004.

52. Turner JR: Molecular basis of epithelial barrier regulation: From basic mechanisms to clinical application. Am J Pathol 169: 1901-1909, 2006.
53. Takeichi M: Cadherin cell adhesion receptors as a morphogenetic regulator. Science 251: 1451-1455, 1991.

54. Tsai HT, Lee TH, Yang SF, Lin LY, Tee YT and Wang PH: Markedly elevated soluble E-cadherin in plasma of patient with pelvic inflammatory disease. Fertil Steril 99: 490-495, 2013.

55. Reiss K,Ludwig A and Saftig P: Breaking up the tie: Disintegrin-like metalloproteinases as regulators of cell migration in inflammation and invasion. Pharmacol Ther 111: 985-1006, 2006.

This work is licensed under a Creative Commons Attribution-NonCommercial-NoDerivatives 4.0 International (CC BY-NC-ND 4.0) License. 